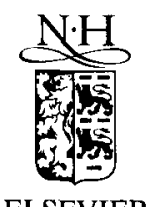

ELSEVIER

\title{
On the origin of second-peak splitting in the static structure factor of metallic glasses
}

\author{
Benjamin W. van de Waal * \\ Department of Physics, University of Twente, PO Box 217, 7500 AE Enschede, The Netherlands
}

Received 22 April 1994; revised 7 November 1994

\begin{abstract}
It is proposed that the splitting of the second peak of the total static structure factor, $S(k)$, of many metallic glasses is essentially the same feature as the indentation at $k \sigma=(9 / 2) \pi$ in the function $\left(\sin k \sigma+\alpha^{-1} \sin k \alpha \sigma\right)$, caused by the coincidence of the fourth minimum of the second term with the third maximum of the first term when $\alpha \approx 5 / 3$. Together with the strong-weak relation of the split peak components of $S(k)$, this feature indicates the splitting to be direct evidence for face-sharing of regular tetrahedra $(\alpha=2 \sqrt{2 / 3})$ dominating the topological short range order; increasing the number of face-sharing tetrahedra in local structural units indeed increases the amount of peak splitting in $S(k)$; a dense random packing of well defined identical structural units (DRPSU), with neighbouring units linked together by a shared icosahedron, is described in detail. The packing fraction in a homogeneous, isotropic 1078-atom model is 0.67 , after static relaxation under a two-body Lennard-Jones potential.
\end{abstract}

\section{Introduction}

A characteristic feature in the total static neutronor X-ray structure factor, $S(k)$, (or the partial Bhatia-Thornton density-density structure factor, $\left.S_{\mathrm{NN}}(k)\right)$ of many binary metallic glasses is the splitting of (or shoulder on) the second peak, at $k \sigma \approx$ $(9 / 2) \pi$, in a (usually) larger lower- $k$ and a smaller higher- $k$ component ( $\sigma$ is the average effective atomic diameter). The splitting is most pronounced in simple-metal glasses, particularly when the size difference between the atoms is not large (as, for example, in $\mathrm{Ca}-\mathrm{Zn}$ ), but frequently absent in transition metal-metalloid amorphous alloys, such as $\mathrm{Ni}-\mathrm{B}$

\footnotetext{
* Corresponding author. Tel: $+31-53893$ 106. Telefax: $+31-53$ 342 657. E-mail: h.j.w.Zandvliet@tn.twente.nl.
}

[1]. Binary alloys of a late and an early transition metal are intermediate in this respect, as indicated, for example, by the gradual disappearance of the splitting in the series $\mathrm{Ni}-\mathrm{Nb}, \mathrm{Ni}-\mathrm{Zr}, \mathrm{Ni}-\mathrm{Y}$ [2].

Nearly all detailed information on the internal structure of these glasses is derived from three-dimensional models that reproduce the experimentally observable one-dimensional function, $S(k)$, or its Fourier transform, $G(r)$ [3]. Thus, the structure of simple-metal glasses is believed to be rather well modelled by the dense random packing of hard spheres (DRPHS), whereas in metal-metalloid glasses trigonal prismatic structural units are probably the dominant feature in the (otherwise disordered) arrangement [4].

It is the purpose of this paper to demonstrate that a dense random packing of structural units (DRPSU) can model the single and two-component simple- 
metal glasses as well, if the topological short range order (TSRO) is primarily chosen to reproduce the second-peak splitting in $S(k)$; obviously, an explanation of the splitting in terms of TSRO is a prerequisite.

We will concentrate on monatomic (single component) glasses, to avoid complications that may arise from chemical short range order or TSRO connected with size differences, different atomic scattering factors, and because the observed structure factors (and hence the inferred structures) are qualitatively not significantly different from that of the simple binary glasses; a characteristic example, obtained with cold vapour deposition [5], is reproduced in Fig. 1.

\section{Structure factor and topological short range order}

The derivation of local structure in metallic glasses from the diffraction experiment requires an interpretation of the pair distribution function (PDF), $g(r)$, or the reduced radial distribution function, $G(r)=$ $4 \pi \rho_{0} r[g(r)-1]$, derived from the observed structure factor, $S(k)$, by evaluating the Fourier sine transform of the reduced interference function $F(k)$ $=k[S(k)-1], G(r)=(2 / \pi) \int F(k) \sin k r \mathrm{~d} k$, in terms of atomic arrangements. However, poor resolution of the PDF, especially in the range $1.5 \lessgtr r \lessgtr 2.0$ (in units of the first peak position $r_{1}$ ), which possibly reflects distortions from ideal of the structural units when they are packed together in space, prevents a straightforward interpretation.

The situation is quite different in the network glasses, like the amorphous semiconductors, a-Ge and $\mathrm{a}-\mathrm{Si}$, and, in particular, vitreous $\mathrm{SiO}_{2}$, where large bond-length and bond-angle distortions are energetically unfavourable, making the structural units deviate only moderately from their crystalline counterparts. Accordingly, the first two maxima in the reduced $\mathrm{RDF}$ of $\mathrm{a}-\mathrm{SiO}_{2}$ are well-defined, and can be unambiguously assigned to the center-to-corner and the corner-to-corner distance, $r_{1}$ and $2 r_{1} \sqrt{2 / 3}$, respectively, in a regular tetrahedron, apparently adequately describing the local structural arrangement. The contribution of these two peaks to the reduced (neutron) interference function can be approximated

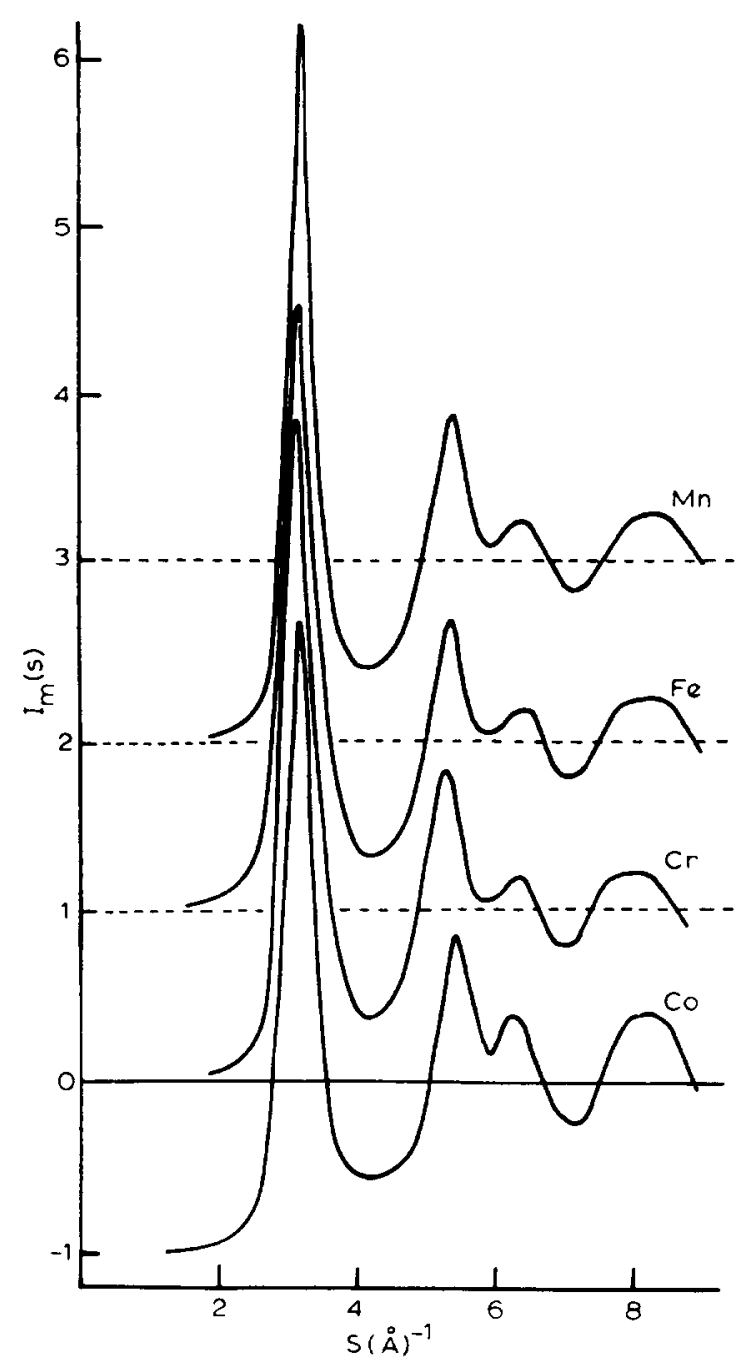

Fig. 1. Interference functions from amorphous films of manganese, iron, chromium and cobalt $(s=(4 \pi / \lambda) \sin (\theta / 2)$; reproduced from Ref. [5], with permission).

by the inverse transform, $F^{\prime}(k)=\int G^{\prime}(r) \sin k r \mathrm{~d} r$, of the modified reduced RDF, $G^{\prime}(r)$, which involves only two Gaussians, fitted to these peaks. The residual $R(k)=F(k)-F^{\prime}(k)$ is found to be appreciable only in the small- $k$ region, up to and including the main peak at $k r_{1} \approx(5 / 2) \pi$ [6]. This remains true when the (narrow) Gaussians are replaced by $\delta$-functions (which only eliminates a small damping, whose effect is negligible in the $k$-range of interest), and applies a fortiori when $S(k)$ s are compared, rather than $F(k)$ s. Thus, in the case of vitreous $\mathrm{SiO}_{2}$, the 


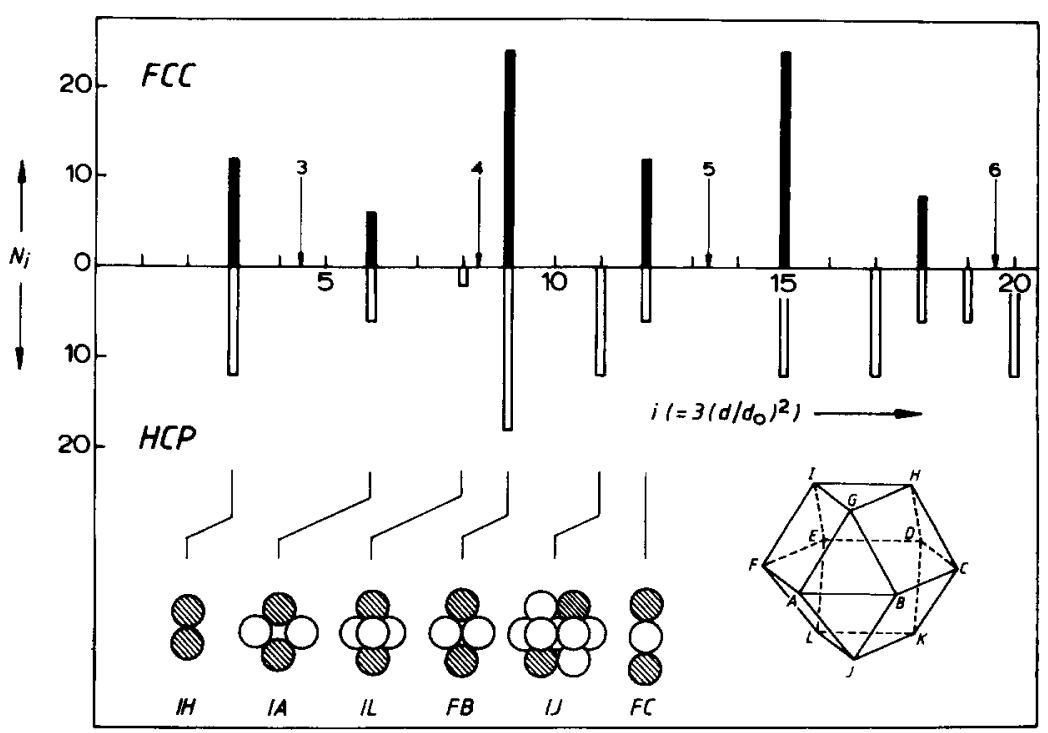

Fig. 2. Idealized radial distribution functions $N\left(d_{i}\right)=N_{i}$ for fcc (filled bars) and hcp (open bars) close packings, for which the interatomic distances can be written as $d_{i}=d_{0} \sqrt{i / 3}, i$ integer. The local atomic arrangements that give rise to some of these distances are indicated. Numbered arrows mark distances for which $d / d_{0}=(4 n-1) / 9$; the $n=4$ arrow is closest to some RDF peaks.

shape of the interference function beyond the main peak is rather well reproduced by a sum of two sines, $n_{1} \sin k r_{1}+n_{2} \sin k r_{2}$, with $r_{2} / r_{1}=2 \sqrt{2 / 3}$.
In particular, the splitting of the second peak, which is also a prominent feature in the interference function of many network glasses, can now be seen to
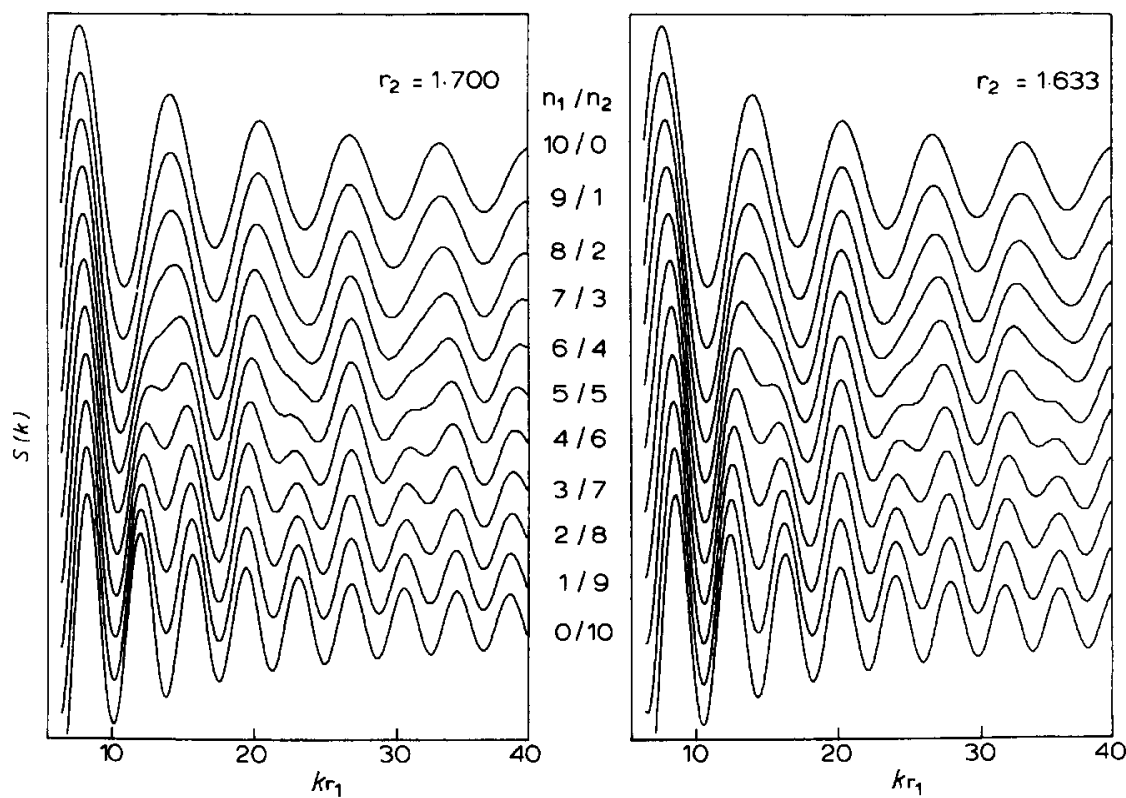

Fig. 3. Plot of $n_{1}\left(\sin k r_{1}\right) / k r_{1}+n_{2}\left(\sin k r_{2}\right) / k r_{2}$ for two values of $r_{2} / r_{1}$, close to $5 / 3$, and different values of $n_{1} / n_{2}$. The shoulder on the second peak, which becomes more pronounced as $n_{1} / n_{2}$ decreases, is on the low- $k$ or high- $k$ side, depending on whether $r_{2}$ is shifted up or down, respectively, from the value $5 / 3 r_{1}$. 
originate in the near-coincidence of the fourth minimum of the second term with the third maximum of the first term.

We want to investigate whether a similar explanation can be given for the second-peak splitting in the structure factor of monatomic metallic glasses, whether this explanation suggests a structural unit that adequately models the topological short range order, as reflected by the appearance of the structure factor beyond the main peak, and whether these structural units can be linked together in a dense, extended, but random array, without inflicting too much distortion on the local order.

Obviously, coincidence of the $n$th minimum of sin $k r_{2}$ with the third maximum of sin $k r_{1}$ requires $r_{2} / r_{1}=(4 n-1) / 9$. Since the local structure of the amorphous metals is likely to be related to local arrangements in the fcc and hcp crystalline counterparts, it is of interest to relate the values of $r_{2}$ for $n=3,4$, etc., with the RDFs of these structures, cf., Fig. 2. The value for $n=4$ (1.667) is seen to be close to and intermediate between interatomic distances $d_{8}$ and $d_{9}\left(d_{\mathrm{i}}=d_{0} \sqrt{i / 3}\right)$, usually associated with the low-r component of the split second peak of the PDF of the glass.

The effect of slightly shifting the value of $r_{2} / r_{1}$ to $1.667 \pm 0.034$ (the lower value coinciding with $d_{8}=2 \sqrt{2 / 3}$ ) on the contribution to $S(k)$ (rather than to $F(k)$ ), and of varying the ratio $n_{1} / n_{2}$ is shown in Fig. 3. Clearly, in order to have a largesmall relation between the low- and high-side components of the split second peak, $r_{2} / r_{1}$ should be smaller than 1.667 , i.e., corresponding most probably to $d_{8}$; moreover, $n_{2}$ should at least equal $n_{1}$, in order to bring out the splitting clearly, i.e., the total count $N_{\dot{8}}$ of interatomic distances $d_{8}$ in the structural unit should approximately equal the total count $N_{3}$ of $d_{3}$ (it should be remembered that the actual contribution of the term $N_{8}\left(\sin k d_{8}\right) / k d_{8}$ will be smaller, as a result of the larger damping connected with the distortion-broadening of the $d_{8}$-peak in the PDF). The third peak remains unaffected, because the maxima of $\sin k r_{1}$ and $\sin k r_{2}$ coincide; on the other hand, in the case of $r_{2}=1.700$, the shape of the third peak is strongly affected by the near-coincidence of maxima and minima.

Thus, it appears natural to identify, in experimentally or model-derived PDFs of monatomic amor- phous structures, the small- $r$ component $\left(r_{2}\right)$ of the split second peak with $d_{8}(2 \sqrt{2 / 3})$, rather than with $d_{9}(\sqrt{3})$, since an appreciable contribution of $d_{9}$ would reverse the strong-weak relation in the second peak of $S(k)$ (as indeed occurs on crystallization). Accordingly, second-peak splitting in $S(k)$ can be explained from, and is direct evidence for facesharing of regular tetrahedra to dominate the short range order. Also, the large- $r$ component $\left(r_{3}\right)$ in the PDF may be identified with the largest interatomic distance (1.99) in four face-sharing tetrahedra not having a common edge (seven-atom Boerdijk spiral), rather than with $d_{11}(1.91)$ or $d_{12}(2.00)$. It should be noted that, although $r_{3}$ and $r_{2}$ are now seen to result from the same structural motif, it is not the splitting of the second peak of $g(r)$ in $r_{2}$ and $r_{3}$ that is required to produce the splitting in the second peak of $S(k)$; as is clear from Fig. 3, the second-peak splitting of $S(k)$ is (nearly) exclusively attributable to $r_{2}$; inclusion of a third term $n_{3} \sin \left(k r_{3}\right) / k r_{3}$ with $r_{3} / r_{1} \approx 2$ has little effect beyond the first maximum when $n_{3} \precsim n_{2}$. The (relative) absence of interatomic distances between about 1.75 and 2.00 in the PDF can now be explained as follows. The PDF of a simple liquid suggests that each atom is surrounded by a number of more or less well defined shells. Since the distance $\sqrt{2}$ is virtually absent in the liquid, as well as in the glass, it is reasonable to assume that the first-shell atoms are arranged more like an icosahedron than like a cuboctahedron. Second-shell atoms will prefer to sit in tetrahedral pockets over the triangular faces of the first-shell icosahedron (distance $2 \sqrt{2 / 3}$ to central atom), occasionally on the saddles between two neighbouring edge-sharing pockets (distance $\sqrt{3}$ ), but rarely on top of first-shell atoms (distance 2.0), thus practically ruling out positions that are intermediate between 1.75 and 2.00 in the glass. Nevertheless, the distance 2.0 is prominent in the PDF of the glass, and must have a different origin, as indicated already. Indeed, it is the distance between a second-shell atom in a firstshell pocket to one of the first-shell atoms surrounding (but not defining) the pocket. Since this picture assumes some coherence between the spatial orientations of (parts of) the first and second coordination shells in the glass, it is clear that the disappearance of the second-peak splitting in the PDF of the liquid could reflect the loss of orientational coherence be- 
tween first and second coordination shells.

Finally we note that face-sharing of tetrahedra, as a characteristic feature of metallic glass structure, is now derived directly from experimental observations, and not from the analysis of models that happen to reproduce more or less faithfully those observations. This distinction is somewhat in the same spirit as that between crystal structure solution from experimental data and crystal structure prediction, based on assumed interaction models, in crystallography.

\section{The structural unit}

A value of $N_{8} / N_{3} \approx 1$ requires a minimum number of some 25 atoms (as compared with only five atoms in the centered $\mathrm{SiO}_{4}$ tetrahedra, that suffice to give $N_{8} / N_{3}=1.5$ ) in the structural unit (SU), as illustrated in Fig. 4, where different polytetrahedral aufbau-sequences are explored. A sequence is obtained by adding new atoms one-by-one in such a way so as to maximize $N_{8} / N_{3}$ (rather than maximizing the cohesive energy, as was done, for example, by Hoare and $\mathrm{Pal}$ [7] to find plausible structures for isolated clusters). Different sequences are possible, since a less favourable step (with respect to the criterion) may result in a 'better' arrangement in a

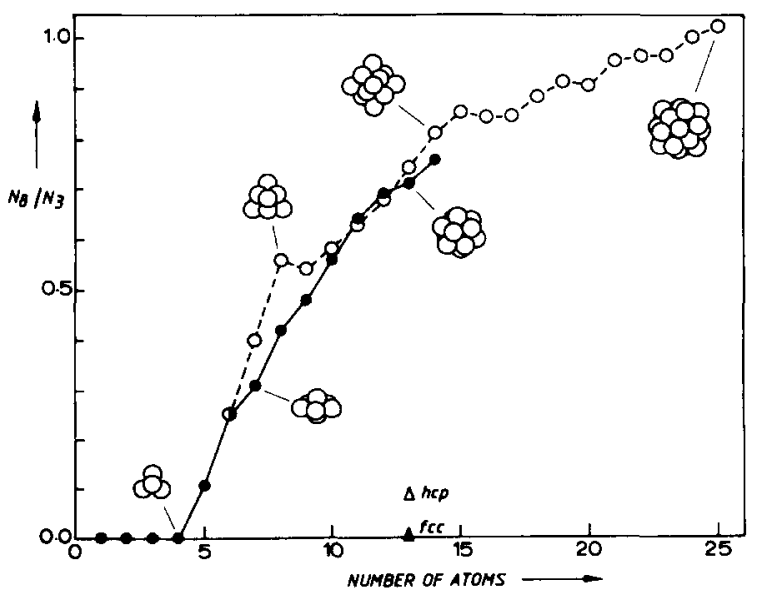

Fig. 4. $N_{8} / N_{3}$ as a function of size of small clusters that have been devised to maximize the frequency $N_{8}$ of the distance $d_{8}=d_{0} \sqrt{8 / 3} .0$, icosahedral sequence. $\bigcirc$, tetrahedral sequence.

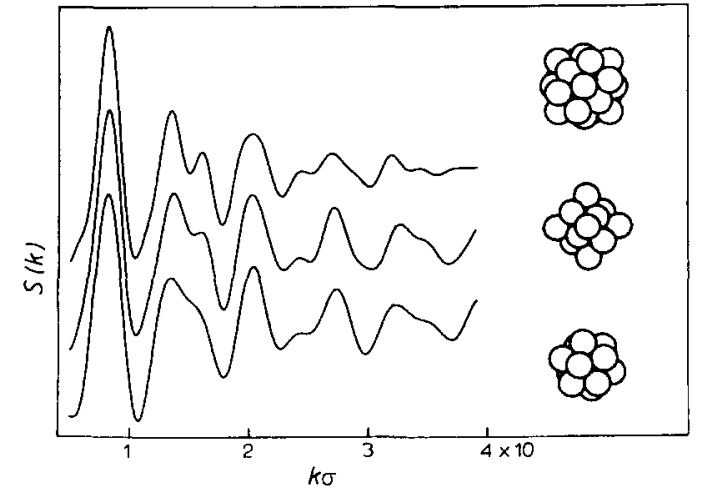

Fig. 5. Structure factor, $S(k)$, for three clusters (also shown in Fig. 4), demonstrating the increased splitting of the second peak with increased value of $N_{8} / N_{3}$ (cf., Fig. $4 ; \sigma$ is the hard sphere diameter).

later stage of the sequence. Thus, an icosahedral sequence (filled circles) and a tetrahedral sequence (open circles; 'tetrahedral' refers to the overall symmetry of the clusters) are competing in this respect. However, beyond $n=14$, the icosahedral sequence can be continued in numerous ways, one of which results in the same 26-atom cluster that is produced by the tetrahedral sequence and which will be shown to be the 'heart' of the SU. Before describing this arrangement in detail, it is of interest to verify that increasing the ratio $N_{8} / N_{3}$ (and at the same time introducing other distances than $d_{3}$ and $d_{8}$ as well) indeed increases the amount of second-peak splitting. This is demonstrated in Fig. 5, where the structure factors, calculated with the Debye formula $\Sigma$ (sin $\left.k r_{i j}\right) / k r_{i j}$, of three different clusters are compared; in passing, we note that second-peak splitting is not necessarily an indication for the frequent occurrence of icosahedra: although the 14-atom structure in Fig. 5 is quite different from an icosahedron, its structure factor clearly exhibits the splitting. Fig. 6 compares the structure factors of two equal-sized clusters, one (fcc) crystalline, the other non-crystalline with $N_{8} / N_{3}$ maximized; the reversal of the strong-weak relation between the components of the split second peak in the crystalline arrangement (which can be identified with the incipient $220 / 311$ splitting in the fcc pattern) is connected with the larger frequency of occurrence of the distance $d_{9}$ (rather than $d_{8}$ ). 
The structure of the 26-atom cluster can be visualized conveniently by following the tetrahedral aufbau-sequence (Fig. 4): starting with a four-atom (hereafter referred to as 'A-atoms') tetrahedron, an eight-atom cluster is obtained by placing B-atoms in the pockets over the four triangular faces; bridging of the B-atoms, across the A-A bonds, with six $\mathrm{C}$-atoms, produces the 14-atom cluster; finally, the 26-atom cluster is obtained by bridging the $\mathrm{C}$-atoms across the $\mathrm{A}-\mathrm{B}$ bonds with $12 \mathrm{D}$-atoms. Thus, all atoms in the 26-atom cluster can be considered to occupy corners, edges or faces of a set of nested (i.e., concentric and parallel) cubes, with sides $d_{\mathrm{A}}=$ $1 / \sqrt{2}, d_{\mathrm{B}}=\frac{5}{3} d_{\mathrm{A}}, d_{\mathrm{C}}=d_{\mathrm{A}}+\sqrt{3}, d_{\mathrm{D}}=3 d_{\mathrm{A}}$ (in units of the hard-sphere diameter). The atomic sites are: four non-neighbouring corners ( $\alpha$-corners) of the A-cube (four-atom tetrahedron), the dual four nonneighbouring corners ( $\beta$-corners) of the B-cube (eight-atom cluster), the six face-centers of the $\mathrm{C}$ cube (14-atom cluster), and the 12 sites on the edges of the D-cube, at a distance $d_{\mathrm{A}}$ from the four $\alpha$-corners of the D-cube (26-atom cluster).

The 26-atom cluster has been described previously by Farges et al. [8]; it is a tetrahedral arrangement of four interpenetrating 13-atom icosahedra, with their centers at the four A-sites, and their threefold axes running through the body center of the A-cube (the fivefold axes run through the edges of the A-tetrahedron, cf., fig. 3 of Ref. [8]). In this arrangement, the four icosahedra have the central

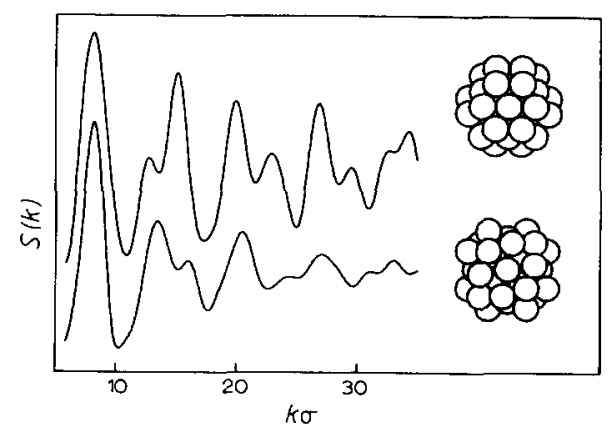

Fig. 6. $S(k)$ for two 38-atom clusters, demonstrating the strongweak inversion of the split second peak components as the second-most important interatomic distance changes from $d_{8}$ $(2 \sqrt{2 / 3})$ in the glass-like structure (lower curve) to $d_{9}(\sqrt{3})$ in the fcc crystalline arrangement (upper curve). (The fcc structure is a two-shell uncentered truncated octahedron; the glass-like structure is shown in more detail in Fig. 7; peak-positions of an infinite fcc crystal would be at $\left.(k \sigma)^{2}=2 \pi^{2}\left[\mathrm{H}^{2}+\mathrm{K}^{2}+\mathrm{L}^{2}\right]\right)$.
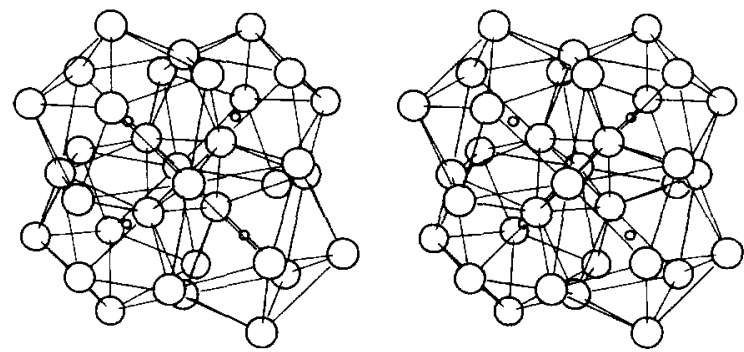

Fig. 7. Stereoview [9] of 38-atom cluster proposed as a structural unit (SU) for a DRPSU model of a monatomic glass. It consists of four icosahedra surrounding a central tetrahedron, similar to the arrangement of oxygens around a central $\mathrm{Si}$ atom in $\mathrm{SiO}_{4}$. The central tetrahedron (of 4 'A-atoms', see text) shares each of its faces with an icosahedron; each icosahedron shares three more faces with the other icosahedra, i.e., there is no interpenetration (shared atoms are ' $\mathrm{C}$-atoms'). The structure has overall tetrahedral symmetry. The central atoms ('B-atoms') of the icosahedra have been drawn small and unconnected, for clarity. Inversion of the central tetrahedron through one of its corners produces 'D-atoms' (drawn mutually unconnected); inversion through 'B-atoms' produces 'E-atoms'. Other icosahedra may be recognized in the interior of the arrangement; thus 'A-atoms' are centra of four interpenetrating icosahedra that share the central tetrahedron. Each of these icosahedra is surrounded by a nearly complete (16 out of 20 atoms) second shell.

A-tetrahedron in common, i.e., the central tetrahedron is inside each of the four icosahedra. Adding another 12 atoms to the sites that are on the face-diagonals of a fifth cube, with side $d_{\mathrm{E}}=4 \frac{1}{3} d_{\mathrm{A}}$, at unit distance from the four $\beta$-comers (i.e., E-atoms bridge D-atoms across B-C bonds), completes the 38-atom structure of Fig. 7 (also shown in Fig. 6), that can now be identified as a structural unit SU underlying a DRPSU. In this SU, the central tetrahedron is outside the four icosahedra, since now they have their centers in the B-atoms (indicated by small dots in Fig. 7). The structure of the 38 -atom SU is quite similar to the centered tetrahedral arrangement of the $\mathrm{SiO}_{4} \mathrm{SU}$ in vitreous $\mathrm{SiO}_{2}$, with icosahedra replacing the oxygens, and a tetrahedron replacing the $\mathrm{Si}$ atom; each of the four icosahedra shares three triangular faces with the other icosahedra, and a fourth face with the central tetrahedron [10]. The similarity with $\mathrm{SiO}_{4}$ allows the linking of SUs in a space filling random network.

\section{Extended models}

An extended structural model of a monatomic glass can now be obtained by randomly packing 

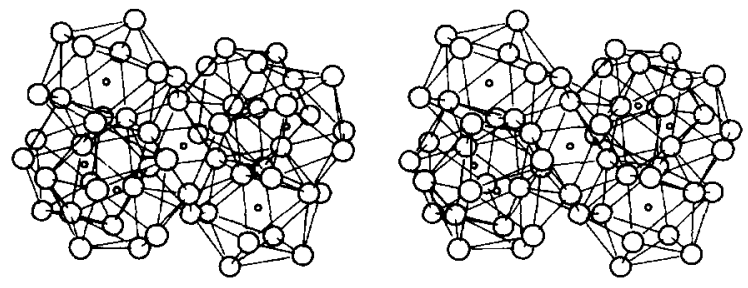

Fig. 8. Linking of SUs is accomplished by sharing an icosahedron. This structure is similar to that of a $\mathrm{Si}_{2} \mathrm{O}_{7}$ fragment in $\alpha$-quartz (the oxygens would be centered at the small dots). Eventually, each icosahedron will share faces with six other icosahedra (as does the central icosahedron in this drawing), and is surrounded by a shell of 20 atoms, approximately in tetrahedral sites over triangular faces.

together the SUs that have been obtained in the preceding section by requiring short range order to be consistent with the shape of the structure factor beyond the main peak. Apparently, the SUs will become distorted by the packing, and new interatomic distances, between atoms in different SUs, come in, possibly decisively modifying the PDF and the small- $k$ region of the structure factor. Moreover, in general, the packing together of SUs will introduce voids, causing the overall density to be significantly below the observed density. The question whether these implications of extending the structure to a size, that can be considered representative for an experimentally observable system, can invalidate the entire procedure, can only be answered by actually constructing and relaxing an extended model, and calculating its structure factor. Thus, Figs. 8-11 illustrate the different steps in the assemblage of a larger cluster from a limited number of SUs. Fig. 8 shows that the linking of two SUs is accomplished
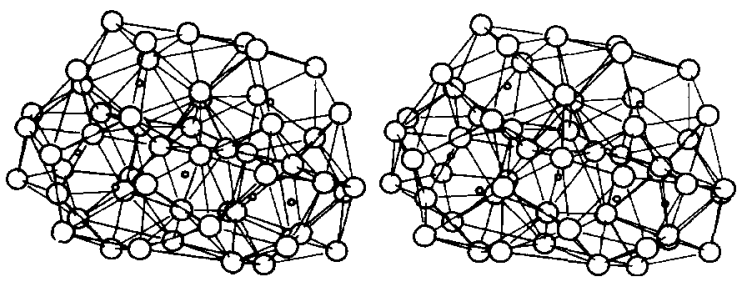

Fig. 9. Limited randomness in the linking of SUs can be introduced by rotating one $\mathrm{SU}$ about a symmetry axis of the shared icosahedron ( $72^{\circ}$ about a fivefold axis, in this example). The structure has been relaxed under a LJ potential, after overlapping atoms have been removed.
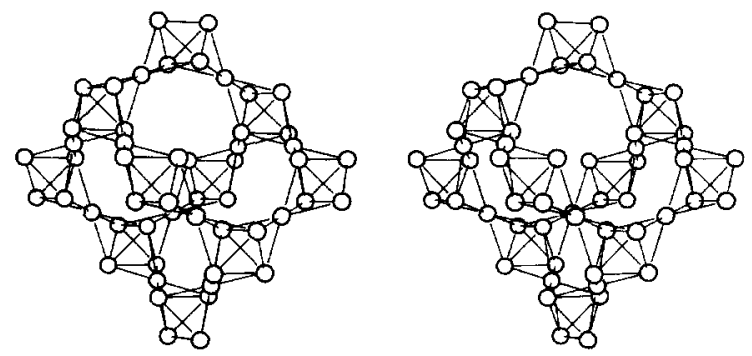

Fig. 10. Frame of the 105-atom cluster of Fig. 11. It is obtained by placing four-atom tetrahedra at the carbon positions of an adamantane fragment of the diamond lattice, and single atoms at the midpoints of the $\mathrm{CC}$ bonds. The latter will be the centra of icosahedra that share faces with the tetrahedra. The closed loops facilitate a systematic search for regions with unrealistic (high or low) density.

by the sharing of an icosahedron, similar to the sharing of an oxygen atom by two $\mathrm{SiO}_{4}$ units in a $\mathrm{Si}_{2} \mathrm{O}_{7} \alpha$-quartz crystal fragment (the small dots in Fig. 8 would correspond to the oxygen positions). Randomness in the interconnection of the units can be introduced by rotating one SU about one of the symmetry axes of the shared icosahedron, while keeping the other SU fixed in space; an example is shown in Fig. 9. Apparently, the number of dimer configurations is limited, by contrast with that of the $\mathrm{Si}_{2} \mathrm{O}_{7}$ moiety where the torsion- and bond-angles $\alpha$ and $\beta$ are independent and not restricted to sub-multiples of $2 \pi$. Although steric limitations would seem to be a further obstacle (because of the size of the icosahedra, as compared with the size of the oxygen atoms in $\mathrm{SiO}_{4}$ ), the possibility of scavenging the
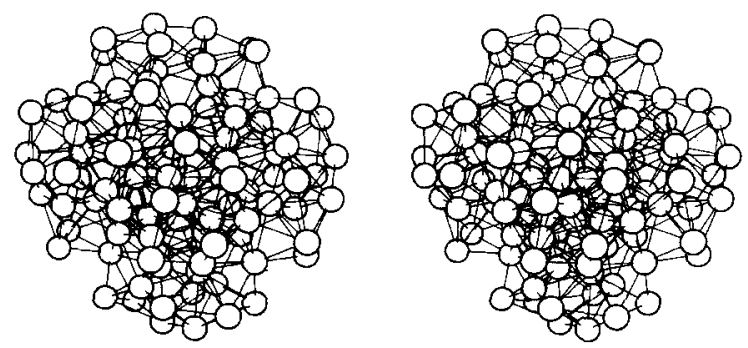

Fig. 11. Finished 105 -atom structure, obtained from the structure of Fig. 10 by completing the icosahedra (by placing ' $\mathrm{C}$-atoms'), introducing an additional atom in the central hole and removing two randomly chosen atoms to reduce the high symmetry. The structure was relaxed under a $\mathrm{LJ}$ potential. 


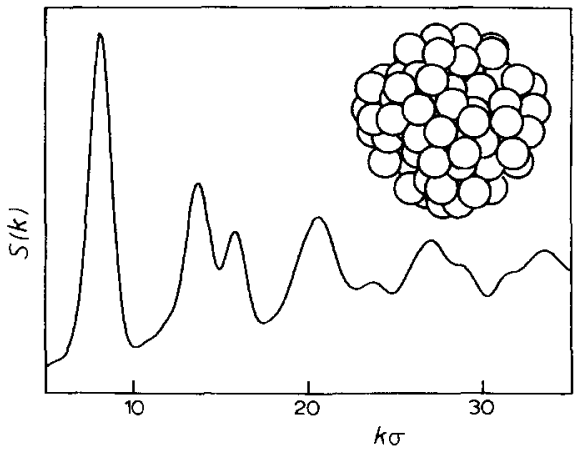

Fig. 12. The structure factor, $S(k)$, of the 105-atom structure shows pronounced second-peak splitting and narrowing of the main peak ( $\sigma$ is the $\mathrm{LJ}$ equilibrium distance: $\sigma=2^{1 / 6} \sigma_{\mathrm{LJ}}$ ).

icosahedra (i.e., removing overlapping atoms) removes this problem.

In order to investigate whether repeated linking of SUs, as in Fig. 8, will produce regions with too low (voids) or too high (overlap) density, SUs were placed on the nodes of an adamantane fragment of the diamond lattice (Fig. 10: only A- and B-atoms shown; Fig. 11: all atoms shown).

The finished structure consists of 12 face-sharing icosahedra and 10 linking tetrahedra. No two atoms are too close, and only in the center of the structure does an octahedral hole allow the insertion of a single additional atom. The structure was relaxed after two randomly chosen atoms had been removed to disrupt the high symmetry. The structure factor for this 105-atom assembly is shown in Fig. 12; the pronounced splitting of the second peak and the over-all appearance indicate that the local order is not severely disturbed by the packing of the SUs in a compact arrangement. Moreover, the narrowing and increased intensity of the main peak indicate that the network topology that results from the packing procedure adequately models the medium range order, although randomness (in the network) has not been introduced explicitly. The construction of Fig. 10 is easily extended in space. Thus, a 1078-atom model was constructed (shown in cross-section in Fig. 13) and relaxed after 10 randomly chosen atoms had been removed from a 1088-atom model. It is rather surprising that, although (so far) only straight bonds have been used (i.e., the assembly of icosahedra is more like the arrangement of the oxygen atoms in a

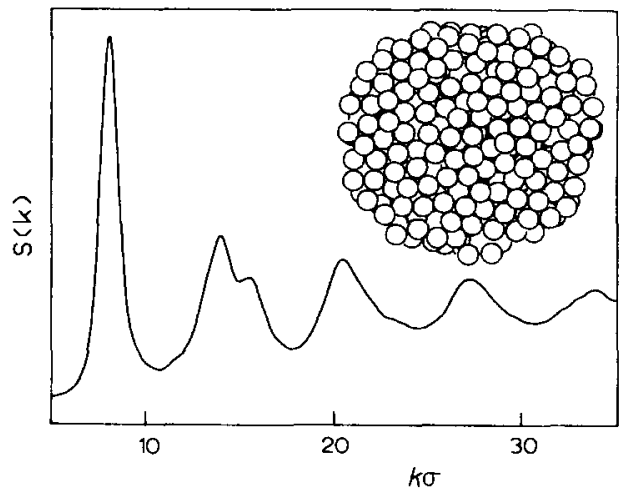

Fig. 13. Structure factor of a 1078-atom model (shown in crosssection, inset), obtained by extending the procedure depicted in Figs. 10 and 11, relaxed after removal of 10 randomly chosen atoms. First-peak sharpening, second-peak splitting and progressive damping of further oscillations are in good qualitative agreement with experimental observations ( $\sigma$ as in Fig. 12).

quartz crystal than in a $\mathrm{SiO}_{2}$ glass), the structure factor (Fig. 13) has all the characteristic features of a glass. Apparently, the random removal of a few atoms, the finiteness of the sample and the static relaxation suffice to suppress effects of lattice periodicity. A comparison of Figs. 5, 6, 12 and 13 shows that the first peak becomes higher and sharper as the size of the sample is increased. At the same time, the amount of splitting of the second peak is reduced in

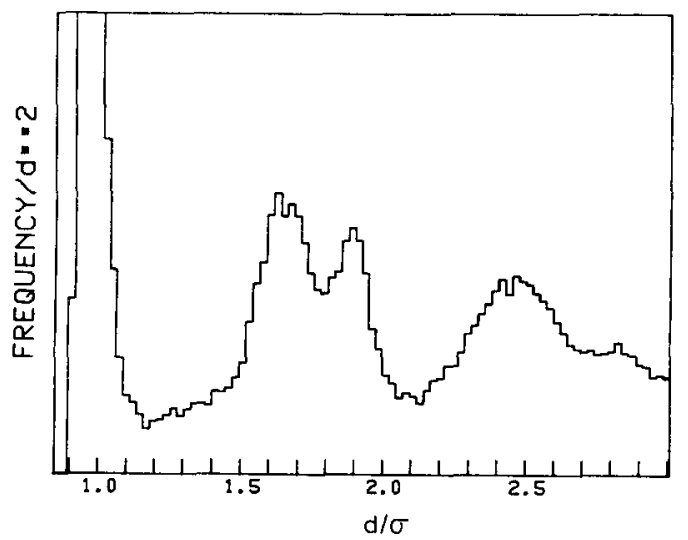

Fig. 14. Frequency of occurrence of the two-particle distance, $d$, divided by $d^{2}$, for the 1078 atom model. This is comparable to the PDF $g(r)$ for distances that are small compared with the cluster radius. The curve is in good qualitative agreement with PDFs derived from experimental data ( $\sigma$ as in Fig. 12). 
the large sample, conceivably because of further broadening of the $d_{8}$ peak in the RDF. The PDF is shown in Fig. 14; it too shows all the characteristic features of a glass, notably a splitting of the second peak (as compared with the liquid), and the absence of a $d_{6}(\sqrt{2})$ peak (as compared with the crystalline solid). The first peak has its maximum at $d=0.958$ (in units of the Lennard-Jones dimer equilibrium distance, $\sigma=2^{1 / 6} \sigma_{\mathrm{LJ}}$ ), the average first neighbour distance (taking the first minimum at $d=1.2$ ) is 0.965 . In units of this average, the split second peak components are at 1.72 and 1.96 , respectively. The value 1.72 is surprisingly large, in view of our efforts to maximize the frequency of occurrence of the distance 1.633; it does not reflect frequent occurrence of the arrangement, FB, in Fig. 2, however, as is apparent from an examination of the structure. The origin may be in the use of a 6:12 $\mathrm{LJ}$ potential [11] for the relaxation, whose relatively hard repulsive part tends to shift the first component outward [12]; indeed, it was found that relaxation significantly reduces the amount of second peak splitting in $S(k)$. Also, the free boundary affects the position of the PDF peaks: omitting 120 surface atoms from the distance statistics shifts the second peak (at 1.72) to the left. The packing fraction is 0.648 within a sphere containing 1033 (of 1078) atoms, and 0.672 within a sphere with 955 atoms, taking $\sigma=0.96$; reducing the sphere radius does not increase this number any further, reflecting a remarkable homogeneity. The LJ cohesive energy per atom is $6.68 \epsilon$, as compared with $7.11 \epsilon$ for a spherical fcc crystal of equal size, and with $8.61 \epsilon$ for an infinite fcc crystal at $0 \mathrm{~K}$.

Crystallization. The detailed description of the model allows some speculation as regards the question what happens when the glass crystallizes. Since the idealized structure consists of face-sharing icosahedra and tetrahedra that are all virtually equivalent, it will be necessary to introduce defects that can act as centers for crystal nucleation, e.g., bent network bonds, incomplete icosahedra, double icosahedra, etc. A defect that is of particular interest in this connection is shown in Fig. 15. It consists of the intergrowth of two (incomplete) Ino's decahedra, i.e., icosahedra, in which two parallel pentagonal rings have been rotated $18^{\circ}$ in opposite directions, resulting in five square faces replacing 10 triangular

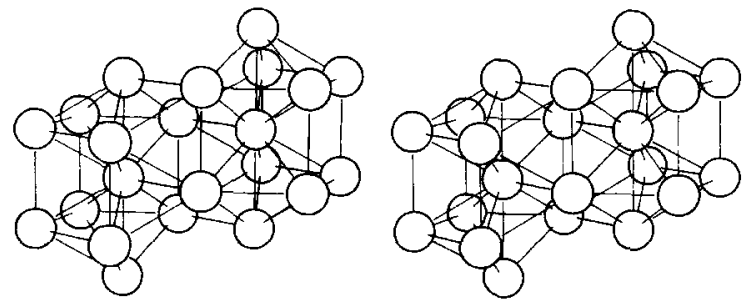

Fig. 15. 22-atom cluster, obtained by fusion of two incomplete (11-, rather than 13-atom) Ino's decahedra (icosahedra, with two parallel pentagonal rings rotated $18^{\circ}$ in opposite directions). The cluster surface exhibits sites with three-, as well as fourfold coordination. Occupation of the latter with new atoms produces new fourfold sites, and will eventually result in a (nearly perfect) fec crystal. The arrangement could trigger crystallization in a glass. (Note the trigonal prisms near the center of the arrangement.)

faces in the original icosahedron. In Fig. 15 two such decahedra (actually: pentadecahedra) have been fused after removal of four atoms (two from each decahedron), to give a 22 -atom cluster. The atomic arrangement in this cluster is the same as that found in the crossing region of intersecting stacking faults in (otherwise perfect) fcc crystals. If such clusters are used as seeds for sequential building algorithms [13], they will not produce a glass but rather a (nearly perfect) fcc crystal, provided new atoms are placed in sites with fourfold coordination, rather than tetrahedral sites [14]. If loops, such as those visible in Fig. 10, are a significant feature in the glass structure, loop-closing mismatch could produce local arrangements such as that of Fig. 15. Mismatch of the 22-atom cluster with its environment could create some space for atomic mobility, necessary for atoms in tetrahedral sites to move over to more favourable sites on the surface of the crystal nucleus. Since the crystal has the higher density, this mobility will increase as the crystal grows.

\section{Discussion}

The explanation of second-peak splitting (rather than stereochemical considerations) has provided guidance for the construction of a structural unit, not only incorporating short range order compatible with the observed structure factor, but also allowing inter- 
connection with neighbouring units, without severe distortions within these units. Even if the final result (a 3D model arrangement of like atoms) would be virtually the same as that obtained by DRPHS model building [12], quench simulation by molecular dynamics [15] or sequential atom addition to a selected seed $[5,13,16]$, the DRPSU approach allows a more transparent description, in which different aspects (network topology, interconnection mode, structural unit) can be distinguished and treated separately. Moreover, other properties are easily recognized without the need to perform statistical analyses; thus, it is immediately clear that the structure is homogeneous, virtually isotropic and built from tetrahedra whose distortions (inherent to frustration [17], the impossibility to tile flat space with tetrahedra) are evenly distributed. Since it is essentially the frequency of occurrence of face-sharing tetrahedra that produces the second-peak splitting, it is clear that statistical analyses of computer glasses that do not explicitly enumerate such arrangements may severely underestimate the glass character. Thus, Voronoi analysis [18] can give an impression of the number of (virtually) undistorted icosahedra, but not of facesharing tetrahedra not participating in the formation of icosahedra. Illustrative in this respect is that the Voronoi signatures of atoms in local fcc environment are identical to those for atoms in hcp environments, although only in the latter case is face-sharing of tetrahedra present. Interstice distribution analysis seems [19] a good alternative, in view of its potential to give an estimate of the average number of tetrahedral holes within a critical distance (i.e., corresponding to face-sharing) of a central tetrahedral hole. A drawback of this method is the sensitivity of the results to the criteria applied.

Bond type labeling [20] is probably most selective in that it explicitly counts [2331] atom pairs participating in face-sharing tetrahedra. The total count, relative to the total number of atoms, could well distinguish between liquid, fcc solid, hcp solid or glass (with a high count of [2441] distinguishing the solid from the liquid). Although a high [1551] frequency is also indicative of face-sharing, it may result in an underestimate, not counting face-sharing tetrahedra that do not participate in complete pentagonal bipyramids or icosahedra. Moreover, as pointed out, second-peak splitting is due to next- nearest neighbours, whereas the label [1551] refers to nearest neighbours.

So far, the possibility of including bent network bonds in the structure has not been explored. It is more complicated than in the case of $\mathrm{SiO}_{2}$ glasses, since not all torsion-and bond-angles are allowed. Whereas voids in a $\mathrm{SiO}_{2}$ network, which cannot accommodate a single atom, have their counterpart in the present monatomic glass model, these voids can accommodate single atoms (but no complete icosahedra). Thus, a search for voids will be necessary to prevent the packing density from dropping below acceptable limits. However, as is clear from Figs. 13 and 14, additional randomness is not required to produce a 1000 -atom sample having all structural characteristics of a glass, although a further extension will eventually produce features connected with periodicity. However, in this respect the model is not worse than those obtained from MD quench simulations employing periodic boundary conditions.

\section{Conclusion}

Although the icosahedron is an important structural motif in our model glass, it is not primarily this arrangement that is responsible for the second-peak splitting of $S(k)$. Rather it is part of the most efficient local solution to the problem to make as many nearly regular tetrahedra face-sharing as possible. It is the latter requirement which derives directly from the peak-splitting, together with the assumption of non-directional bonding.

Stimulating discussions and correspondence with Dr G. Torchet are gratefully acknowledged.

\section{References}

[1] P.P. Gardner, N. Cowlam and H.A. Davies, J. Phys. F15 (1985) 769; K. Suzuki, T. Fukunaga, F. Itoh and N. Watanabe, in: Rapidly Quenched Metals, ed. S. Steeb and $\mathrm{H}$. Warlimont (Elsevier, New York, 1985) p. 479.

[2] Ch. Hausleitner and J. Hafner, Phys. Rev. B45 (1992) 128.

[3] G.S. Cargill III, Solid State Phys. 30 (1975) 227.

[4] P.H. Gaskell, J. Non-Cryst. Solids 32 (1979) 207. 
[5] P.K. Leung and J.G. Wright, Philos. Mag. 30 (1974) 185, 995; P.K. Leung, J.J. Quinn and J.G. Wright, Philos. Mag. B38 (1978) 127.

[6] A.C. Wright, R.A. Hulme, D.I. Grimley, R.N. Sinclair, S.W. Martin, D.L. Price and F.L. Galeener, J. Non-Cryst. Solids 129 (1991) 213.

[7] M.R. Hoare and P. Pal, Adv. Phys. 20 (1971) 161.

[8] J. Farges, M.F. de Feraudy, B. Raoult and G. Torchet, Surf. Sci. 156 (1985) 370.

[9] Stereoperception is easily achieved without a special viewer, by gazing at the picture, at normal reading distance, allowing both images of the stereo pair to merge, before refocussing. A piece of paper, held vertically between the two drawings (while avoiding unequal illumination), as well as colouring a single atom (in both drawings), can be helpful.

[10] Similar tetrahedral arrangements of icosahedra (around an octahedron, rather than a tetrahedron) are found in crystalline $\mathrm{VAl}_{10}$ [P.J. Brown, Acta Crystallogr. 10 (1957) 133]; however, each two icosahedra share only one (rather than three) atoms.

[11] The $\mathrm{LJ}$ potential is known to give only a poor representation of the interatomic interactions in a metallic glass; it was used only to ensure that the model was not unstable under a simple potential.

[12] J.L. Finney, Nature 266 (1977) 309; in: Structure of NonCrystalline Materials, Proc. Symp. 1976, ed. P.H. Gaskell (TransTech, Aerdermannsdorf, 1977) p. 35.
[13] C.H. Bennett, J. Appl. Phys. 43 (1972) 2727.

[14] B.W. van de Waal, Phys. Rev. Lett. 67 (1991) 3263.

[15] J.N. Cape, J.L. Finney and L.V. Woodcock, J. Chem. Phys. 75 (1981) 2366; M. Tanaka, J. Phys. Soc. Jpn. 51 (1982) 3075, 3802; 52 (1983) 2046; 54 (1985) 2069; 55 (1986) 3108, 3428; T.A. Weber and F.H. Stillinger, Phys. Rev. B31 (1985) 1954; S. Nosé and F. Yonezawa, J. Chem. Phys. 84 (1986) 1803; R.A. LaViolette, Phys. Rev. B40 (1989) 9952; M.S. Watanabe and K. Tsumuraya, J. Chem. Phys. 87 (1987) 4891; 88 (1988) 1991; 92 (1990) 4983; W.C. Swope and H.C. Andersen, Phys. Rev. B41 (1990) 7042; F. Yonezawa, Solid State Phys. 45 (1991) 179; D.W. Qi, S. Wang and R.S. Liu, Phys. Rev. B44 (1991) 884; 45 (1992) 451; 46 (1992) 12001.

[16] T. Ichikawa, Phys. Status Solidi (a)19 (1973) 707; (a)29 (1975) 293; P. Mrafko and P. Duhaj, J. Non-Cryst. Solids 17 (1975) 143; J.F. Sadoc, J. Dixmier and A. Guinier, J. NonCryst. Solids 12 (1973) 46.

[17] D.R. Nelson and F. Spaepen, Solid State Phys. 42 (1989) 1.

[18] J.C. Gil Montoro and J.L.F. Abascal, J. Phys. Chem. 97 (1993) 4211, and references cited therein.

[19] J.L. Finney and J. Wallace, J. Non-Cryst. Solids 43 (1981) 165.

[20] J.D. Honeycutt and H.C. Andersen, J. Phys. Chem. 91 (1987) 4950. 